Nota clínica

\title{
Manejo conservador de los traumatismos renales por arma blanca. Presentación de dos nuevos casos y revisión de la literatura
}

Juan P. Caballero Romeu, Julián Megías Garrigós, Asier Leivar Tamayo, Juan F. Galiano Baena, Carla Pérez Tomás, Enrique Herrero Polo

Servicio de Urología. Hospital General Universitario de Alicante. España

\section{Resumen}

Introducción: El tratamiento de los traumatismos renales por arma blanca ha sido tradicionalmente quirúrgico. Desde hace más 20 años se aboga por un tratamiento conservador con el fin de evitar laparotomías innecesarias y preservar unidades renales. Los criterios para intervenir de forma urgente o las pautas de seguimiento no están establecidos debido a la falta de estudios de calidad.

Material y métodos: Presentamos dos nuevos casos de traumatismo renal por arma blanca en los que se llevó a cabo un manejo conservador y realizamos un revisión de la literatura.

Resultados: Los dos pacientes evolucionaron favorablemente, si bien uno de ellos presentó hematuria durante el postoperatorio inmediato y fue necesaria una embolización selectiva de una fístula arterio-venosa. La mayor parte de los artículos se basan en series de casos o estudios observacionales retrosprospectivos.

Conclusiones: En los traumatismos renales por arma blanca el diagnóstico es clínico y el estadiaje de las lesiones radiológico con una tomografía computarizada. El manejo conservador es de elección cuando el paciente está estable hemodinámicamente y las lesiones no requieren reparación inmediata. Estos pacientes requieren un seguimiento a largo plazo para monitorizar la aparición de complicaciones. Son necesarios estudios de mayor calidad que aporten una mayor evidencia científica.

Palabras clave: Heridas penetrantes. Riñón. Manejo clínico.

Stab wounds of the kidney: conservative management. Two new cases and review of the literature

\begin{abstract}
Introduction: Stab wounds of the kidney have traditionally been managed by open surgery. Nowadays the conservative management of stab wounds injuries is extended in order to avoid unnecessaries nephrectomies and laparotomies without increasing morbidity and mortality. Although there is no a strong evidence to recommend when to operate or what sort of follow up we must do.

Material and methods: We present two new cases of stab wounds injuries managed conservatively. We performed a systematic review of the literature.

Results: Both patients evolved favorably although one of them presented hematuria 7 days after the traumatism and we performed an embolization of an arteriovenous fistula. Most of the articles are based on series of cases or retrosprospective studies.

Conclusions: The diagnosis and staging of stab wounds of the kidney must be done with clinic and CT scan. Conservative management is required when the patient is not hemodinamically unstable and injuries do not require inmediate repair. These patients require a long term follow up to prevent the appearance of complications. Randomized prospective multicenter trials are needed to support the optimum management for each kidney injury degree.
\end{abstract}

Keywords: Stab wounds. Kidney. Case management.

$\mathrm{L}$ os traumatismos renales (TR) representan aproxximadamente del 1 al $10 \%$ de todos los traumatismos, siendo el riñón el órgano genitourinario y de la cavidad abdominal más afectado. Estos traumatismos son más frecuentes en varones de 20 a 30 años de edad.
Las lesiones renales se producen como consecuencia de traumatismos cerrados en el 90-95\% de los casos, los traumatismos renales abiertos (TRA) son menos frecuentes y suelen ser por arma de fuego o por arma blanca. En los medios urbanos la creciente violencia callejera condiciona que 
el porcentaje de heridas por arma blanca, o por arma de fuego, aumente respecto a los traumatis$\operatorname{mos}_{\text {cerrados }}{ }^{1}$.

Los TRA se asocian con daño de otros órganos en el $61-100 \%$ de los casos frente a un 20-65\% de los traumatismos renales cerrados, según las distintas series de la literatura.

Clásicamente los TRA, y los traumatismos abdominales abiertos en general, han sido tratados mediante cirugía abierta, pero, hace más de 50 años, algunos cirujanos pediátricos observaron que determinadas lesiones traumáticas del bazo se podían tratar sin cirugía con buenos resultados, lo mismo ocurrió posteriormente con el manejo de las heridas hepáticas. Esta experiencia, junto al perfeccionamiento de las técnicas de imagen, ha hecho que estas lesiones se manejen de forma conservadora. La calidad de los estudios existentes en la literatura es baja y no permite determinar cuál es el manejo idóneo de los traumatismos renales ${ }^{2}$.

En nuestro centro hemos atendido, en el último año, a dos pacientes con TRA por arma blanca y en ambos casos se decidió un manejo conservador. A continuación presentamos estos casos y una revisión de la literatura acerca del manejo conservador de los TRA.

\section{Caso 1}

Varón de 18 años de edad que ingresa en el Servicio de Urgencias tras sufrir una herida por arma blanca en flanco derecho. El paciente está consciente y orientado, su tensión arterial es 160/80 mmHg. En la exploración presenta una herida incisa y penetrante con un orificio de entrada de $2 \mathrm{~cm}$ a nivel intercostal derecho entre D $10 \mathrm{y}$ D 12, además el murmullo vesicular del hemitórax derecho está disminuido.

La analítica de sangre muestra una hemoglobina de $12,7 \mathrm{~g} / \mathrm{dl}$ y un hematocrito de $38,7 \%$. La coagulación es normal.

Se le realiza una tomografía computarizada (TC) abdominopélvica en la que se aprecia una herida abierta en la parrilla costal derecha que atraviesa el segmento 6 hepático y penetra en la cortical renal alcanzando el seno. La clasificamos como una lesión grado 3 de la AAST. Además presenta un hematoma que ocupa los espacios subhepático, intra y perirrenal (Fig. 1). La vejiga está distendida y llena de coágulos por lo que se le coloca un catéter vesical de 3 vías y lavados continuos.

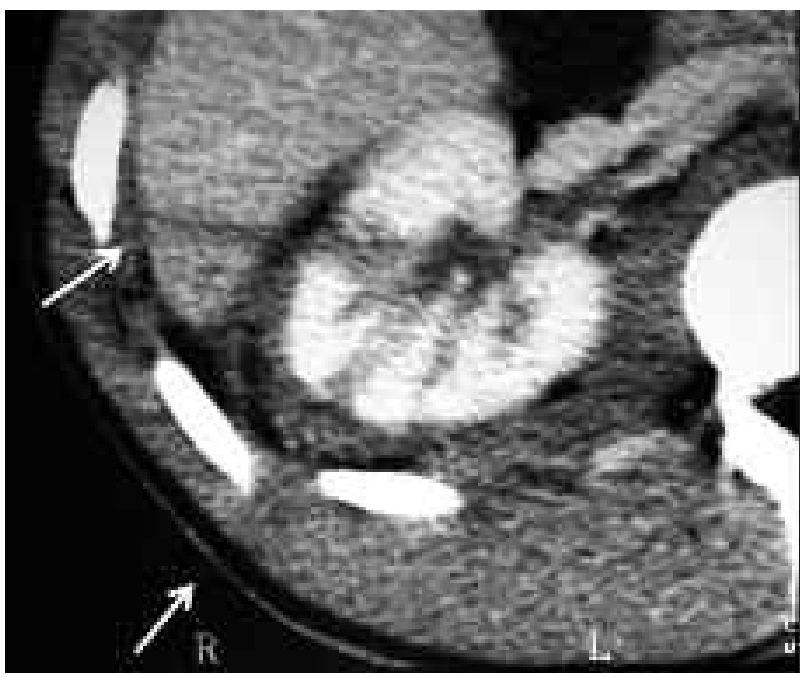

FIGURA 1. La TC muestra el trayecto desde el espacio intercostal derecho hasta el hilio renal con un pequeño hematoma asociado y sin extravasación urinaria. Obsérvese la escasa alteración de los tejidos adyacentes.

El enfermo es valorado por los Servicio de Cirugía y de Urología de forma conjunta y dado que está hemodinámicamente estable se decide una actitud conservadora y el paciente pasa a la Unidad de Reanimación para control.

La evolución fue favorable recuperando el peristaltismo intestinal y la tolerancia oral al cuarto día del ingreso. Una vez desapareció la hematuria se le retiró el catéter vesical.

Al séptimo día del ingreso el paciente presentó dificultades respiratorias por un neumotórax que precisó la colocación de un tubo torácico de drenaje. A las 24 horas de dicho episodio reapareció la hematuria por lo que se realizó una arteriografía diagnóstico-terapéutica. Durante la exploración se objetivó una fístula arteriovenosa intrarrenal en región interpolar que se embolizó con tres coils (Fig. 2). Tras el tratamiento la hematuria remitió y fue dado de alta hospitalaria.

En los controles posteriores con urografía intravenosa (UIV), a los 3 meses y al año, el riñón derecho permanece funcionante en su totalidad y el paciente no presenta complicación alguna (Fig. 3).

\section{Caso 2}

Varón de 37 años de edad acude a urgencias tras ser víctima de una agresión por arma blanca en flanco izquierdo.

El enfermo se mantiene hemodinámicamente estable con una hemoglobina de 15,2 g/dl al ingreso. Valorado por el Servicio de Cirugía Torácica se descartan lesiones intratorácicas. 


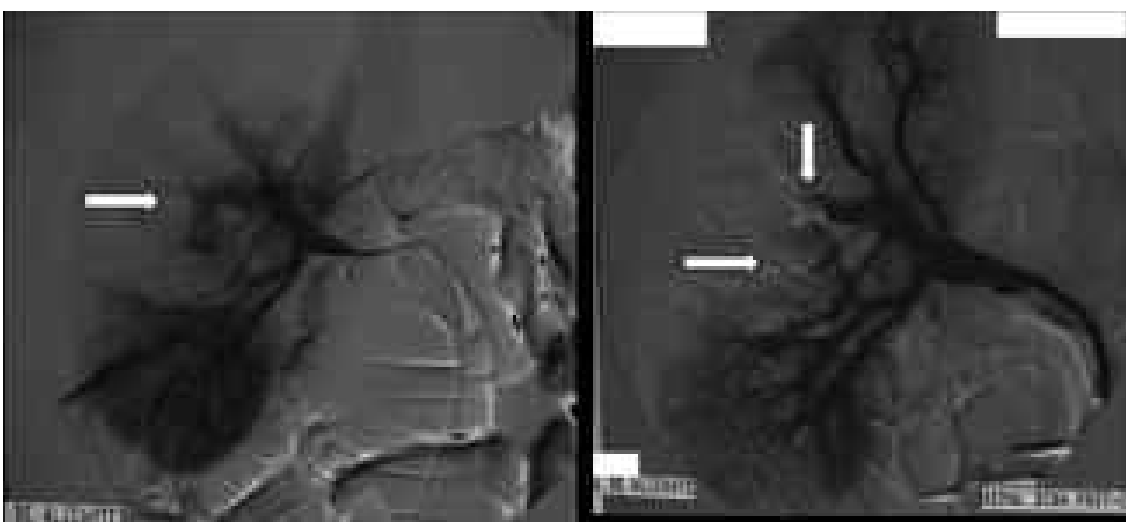

FIGURA 2. La arteriografía permitió localizar los puntos de sangrado y su embolización selectiva con tres coils.
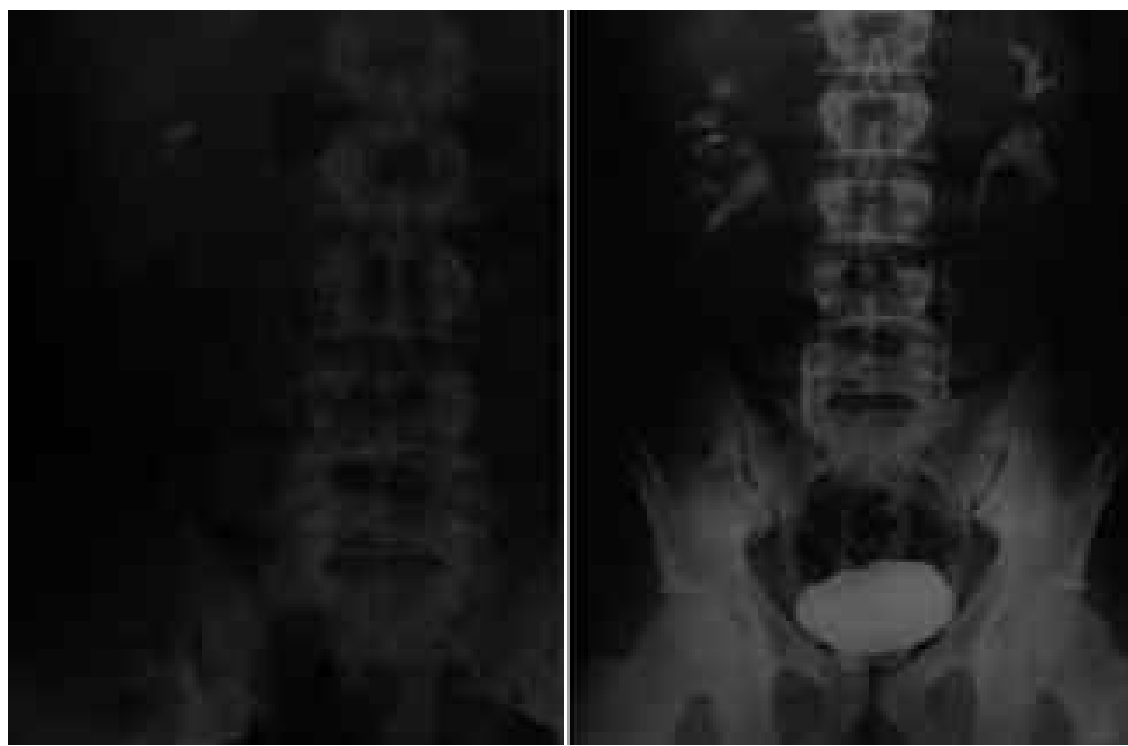

FIGURA 3. En la radiografía simple vemos la posición de los coils y con la urografía intravenosa comprobamos que el riñón capta y elimina el contraste. puntos de entrada y de salida, y la magnitud de la energía del elemento traumático ${ }^{3}$.

Los TRA se pueden clasificar según el punto de entrada ya que esto tiene implicaciones en la evolución del paciente. Si el punto de entrada es posterior a la línea axilar anterior las posibilidades de éxito con un manejo conservador son muy elevadas, en estos casos el daño es principalmente parenquimatoso. En cambio, cuando el punto de entrada es anterior, la posibilidad de daño a nivel del hilio renal es mayor ${ }^{4}$.

La clínica inicial tras un TRA puede incluir: dolor, una tumoración en el flanco, e íleo paralítico. Algunos hallazgos tienen una significación muy variable, la presencia o no de hematuria, así como el grado de la misma, fue propuesto como valor predictivo de cara a una exploración quirúrgica; sin embargo diferentes estudios han demostrado que no existe tal correlación. Actualmente se consideran dos factores principales de cara a una exploración quirúrgica, la estabilidad hemodinámica del paciente y los hallazgos que nos ofrece la TC como sangrado activo o fístulas urinarias ${ }^{5}$.

En los traumatismos renales cerrados la presencia de hematuria puede ser un factor decisivo para solicitar una TC. En cambio, en los TRA la existencia o no de hematuria no es determinante.

Las lesiones renales traumáticas se clasifican según la Organ Injury Scaling of the American Association for the Surgery of Trauma (AAST) (Tabla 1) (1,7. $^{2}$ Según la AAST las lesiones de Grado I y II pueden considerarse como menores. Cuando el traumatismo abdominal únicamente daña el riñón, el 98\% de las lesiones son menores.

Las lesiones de Grado III, IV y V, son consideradas como mayores. El grupo de Federle y Mc Aninch modificó esta clasificación según los hallazgos radiológicos mediante TC (Tabla 2) y actualmente es empleada como referencia en muchos centros ${ }^{8}$.

En los TRA la historia clínica es fundamental para conocer el mecanismo lesional, así como los 


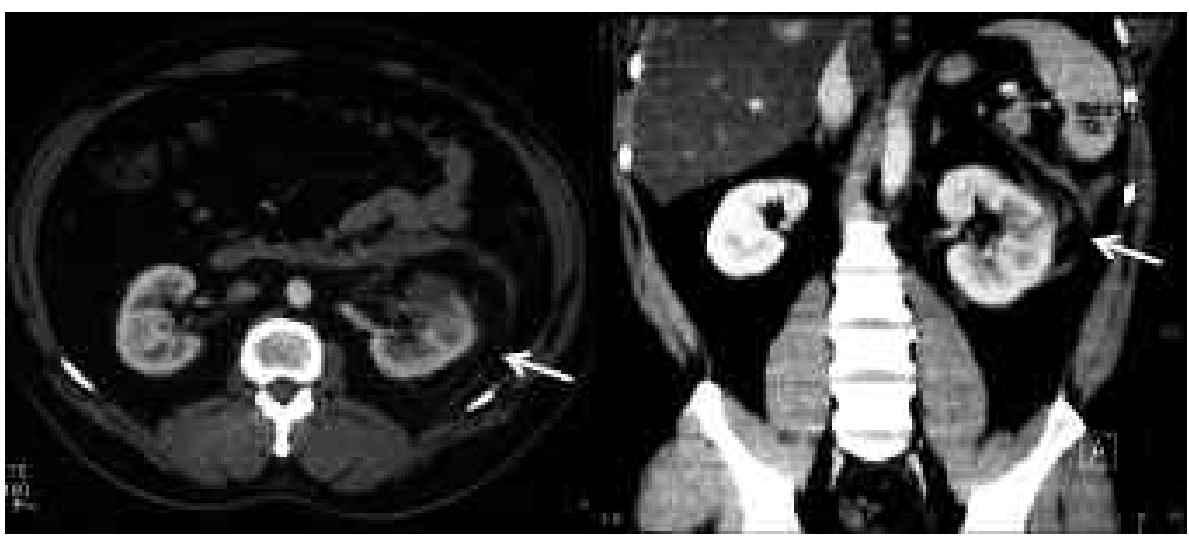

FIGURA 4. La TC muestra una lesión cortical asociada a un hematoma perirrenal bien delimitado.

Tabla 1. Escala de daño renal de la AAST

1. Contusión o hematoma subcapsular no expansivo. No laceración.

2. Hematoma perirrenal delimitado o laceración cortical renal que penetra $<1 \mathrm{~cm}$ en profundidad sin extravasación urinaria.

3. Laceración cortical $>1 \mathrm{~cm}$ sin extravasación urinaria.

4. Laceración renal con extravasación urinaria o afectación arterial o venosa con hematoma contenido.

5. Riñón completamente destrozado o afectación importante del pedículo con avulsión del hilio que desvasculariza el riñón.

Tabla 2. Clasificación de los traumatismos renales por TAC según Federle y col.

\begin{tabular}{|c|c|c|}
\hline Grado & Daño Renal & Descripción \\
\hline 1 & Menor & $\begin{array}{l}\text { a. Contusión renal focal o global } \\
\text { b. Laceración renal superficial } \\
\text { que no afecta al sistema } \\
\text { colector } \\
\text { c. Pequeño hematoma perirrenal } \\
\text { o subcapsular } \\
\text { d. Infarto segmentario isquémico }\end{array}$ \\
\hline 2 & Mayor & $\begin{array}{l}\text { a. Laceración profunda del } \\
\text { parénquima renal que afecta } \\
\text { al sistema colector con } \\
\text { extravasación contenida de } \\
\text { orina } \\
\text { b. Hematoma } \\
\text { perirrenal/subcapsular } \\
\text { moderado o extenso }\end{array}$ \\
\hline 3 & Catastrófico & $\begin{array}{l}\text { a. Afectación del pedículo renal } \\
\text { importante arterial o venoso } \\
\text { b. Fragmentación } \\
\text { parenquimatosa renal con } \\
\text { hemorragia extensa } \\
\text { c. Disrupción de la pelvis renal o } \\
\text { del uréter proximal }\end{array}$ \\
\hline
\end{tabular}

Respecto al tratamiento de las heridas renales abiertas, no existe un consenso sobre la pauta terapéutica a seguir. Algunos autores proponen la exploración quirúrgica de aquellas heridas de alto grado o de las heridas de menor grado pero con lesiones asociadas que requieran una revisión quirúrgica. En cambio otros no cambian el criterio porque haya afectación de otros órganos asociadas ${ }^{9}$.

Algunos autores recomiendan que el manejo de los TRA Grado III dependa de la estabilidad hemodinámica en primer lugar y de los hallazgos de la TC en segundo.

En cualquier caso, es lógico que a mayor grado lesional, mayor es el riesgo de intervención quirúrgica. La frecuencia de intervención según la serie de Santucci y McAninch es según el grado de la AAST: 0\% para lesiones de Grado I, 15\% para el Grado II, 76\% para el Grado III, 78\% para el Grado IV y 93\% para el Grado V.

Las indicaciones absolutas de exploración quirúrgica son la presencia de un hematoma expansivo, pulsátil o no contenido a nivel retroperitoneal o cuando se sospeche la avulsión del hilio renal. La indicación absoluta se define como la pérdida de 3 concentrados de hematíes por día. Son indicaciones relativas: el sangrado persistente, la extravasación urinaria, la no viabilidad del tejido renal o que no se haya podido una estadificación adecuada del traumatismo.

En algunas series se evidencia que en los casos de TRA, cuando se realiza una exploración quirúrgica inmediata, se triplica el riesgo de nefrectomía. Estimamos que ante lesiones de igual severidad la mera exploración quirúrgica condiciona al cirujano, que opta por una actitud más agresiva. El manejo conservador de los TRA permitiría por tanto preservar más unidades renales.

En nuestro caso $\mathrm{n}^{\circ} 1$ la herida renal se asociaba a una herida hepática; sin embargo ni por parte de Cirugía General ni por parte de Urología se creyó conveniente realizar una laparotomía exploradora. Por este motivo opinamos que la generalización del manejo conservador de las heridas abiertas que afectan a órganos sólidos abdominales puede suponer un importante decremento en el número de intervenciones quirúrgicas urgentes. 
Las principales medidas del manejo conservador de las heridas renales abiertas son hidratación, antibioterapia, reposo hospitalario y domiciliario, determinación seriada de hematocrito y la transfusión sanguínea si es preciso. El paciente puede deambular en el momento en que ceda la hematuria guardando únicamente reposo relativo y deben realizarse exploraciones con TC a intervalos según el estado del paciente.

La embolización supraselectiva del parénquima dañado es una buena opción en aquellos pacientes hemodinámicamente estables con una hematuria que no cede con el reposo y que presentan anemización moderada. Con la embolización supraselectiva conseguimos detener el sangrado renal en el 7080\% de los casos. Esta técnica también se emplea cuando secundariamente al TRA se produce una fístula arteriovenosa, como en el caso $n^{0} 1^{10}$.

Cuando sospechemos una fístula arteriovenosa podemos confirmarlo con una ecografía doppler, con un escáner o bien con una arteriografía como exploración mas precisa y que nos servirá para realizar el tratamiento de la fistula.

Por último, no debemos menospreciar la importancia del seguimiento de estos pacientes, ya que a largo plazo pueden desarrollar otro tipo de patologías como la HTA. El seguimiento en las consulta deberá valorar la función y morfología renal con análisis de sangre y pruebas de imagen.

\section{CONCLUSIONES}

La estabilidad hemodinámica es determinante para decidir un manejo conservador en los casos de traumatismo renal abierto. Monitorizaremos la situación clínica y los niveles de hemoglobina. La TC es una herramienta fundamental en la valoración de estos traumatismos.

Las medidas de soporte e incluso las técnicas de radiología intervencionista permiten disminuir laparotomías exploratorias y de este modo conservar unidades renales.

\section{Abreviaturas}

AAST: American Association for the Surgery of Trauma. TC: Tomografía Computarizada

\section{REFERENCIAS}

1. Lynch TH, Martínez-Piñeiro L, Plas E, Serafetinides E, Türkeri L, Santucci RA et al. EAU guidelines on urological trauma. Eur Urol .2005;47(1):1-15.

2. Santucci R. A., Wessells H, Bartsch G, Descotes J, Heyns CF, Mc Aninch JW, et al. Evaluation and management of renal injuries: consensus statement of the renal trauma subcommittee. B J Urol Int. 2004;93(7) 937-954.

3. Cabrera Castillo PM, Martínez Piñeiro L, Álvarez Maestro M, De la Peña JJ. Evaluation and treatment of kidney penetrating wounds. Ann Urol (Paris). 2006;40(5):297-308.

4. Bernath AS, Schutte H, Fernandez R, Addonizio JC. Stab wounds of the kidney: conservative management in flank penetration. J Urol. 1983;129(3):468-470.

5. Kawashima A, Sandler CM, Corl FM, West OC, Tamm EP, Fishman EK, et al. Imaging of renal trauma: a comprehensive review. Radiographics. 2001;21(3):557-574.

6. Alsikafi NF, Rosenstein DI. Staging, evaluation and nonoperative management of renal injuries. Urol Clin N Am 2006;33 (1): 13-19.

7. Kuan JK, Wright JL, Nathens AB, Rivara FP, Wessells H. American Association for the Surgery of Trauma. American association for the surgery of trauma organ injury scale for kidney injury predicts nephrectomy, dialysis, and death in patients with blunt injury and nephrectomy for penetrating injuries. J Trauma. 2006;60(2):351-356.

8. Federle MP, Brown TR, McAninch JW. Penetrating renal trauma: CT evaluation. J Comput Assist Tomogr. 1987;11(6):10261030.

9. Wright JL, Nathens AB, Rivara FP, Wessels H. Renal and extrarenal predictors of nephrectomy from the national trauma data bank. J Urol. 2006;175(3 Pt 1):970-975.

10. de Pablo Cárdenas A, Pinós Paul MA, Jiménez Aristu JI, Iriarte Aristu J, Garrón Aoiz L, Millán Serrano JA. Traumatismo renal severo con tratamiento conservador. Actas Urol Esp. 2005;29 4):436.

Correspondencia autor: Dr. Juan P. Caballero Romeu Servicio de Urología

Hospital General Universitario de Alicante c/ Pintor Baeza $\mathrm{n}^{\circ} 12$ - 03010 Alicante Tel.: 965.93.83.10

E-mail autor: juanpablocaballero@gmail.com Información artículo: Nota clínica

Trabajo recibido: noviembre 2007

Trabajo aceptado: enero 2008 